\title{
HEAVY SUBGRAPHS, STABILITY AND HAMILTONICITY
}

\author{
BINLONG Li ${ }^{a, c}$ AND Bo Ning ${ }^{1 b}$ \\ ${ }^{a}$ Department of Applied Mathematics \\ Northwestern Polytechnical University \\ Xi'an, Shaanxi 710072, P.R. China \\ ${ }^{b}$ Center for Applied Mathematics \\ Tianjin University \\ Tianjin 300072, P.R. China \\ ${ }^{c}$ European Centre of Excellence NTIS \\ 30614 Pilsen, Czech Republic \\ e-mail: bo.ning@tju.edu.cn
}

\begin{abstract}
Let $G$ be a graph. Adopting the terminology of Broersma et al. and Cada, respectively, we say that $G$ is 2-heavy if every induced claw $\left(K_{1,3}\right)$ of $G$ contains two end-vertices each one has degree at least $|V(G)| / 2$; and $G$ is o-heavy if every induced claw of $G$ contains two end-vertices with degree sum at least $|V(G)|$ in $G$. In this paper, we introduce a new concept, and say that $G$ is $S$-c-heavy if for a given graph $S$ and every induced subgraph $G^{\prime}$ of $G$ isomorphic to $S$ and every maximal clique $C$ of $G^{\prime}$, every nontrivial component of $G^{\prime}-C$ contains a vertex of degree at least $|V(G)| / 2$ in $G$. Our original motivation is a theorem of Hu from 1999 that can be stated, in terms of this concept, as every 2-connected 2-heavy and $\mathrm{N}$-cheavy graph is hamiltonian, where $N$ is the graph obtained from a triangle by adding three disjoint pendant edges. In this paper, we will characterize all connected graphs $S$ such that every 2-connected o-heavy and $S$-c-heavy graph is hamiltonian. Our work results in a different proof of a stronger version of Hu's theorem. Furthermore, our main result improves or extends several previous results.
\end{abstract}

Keywords: heavy subgraphs, hamiltonian graphs, closure theory.

2010 Mathematics Subject Classification: 05C38, 05C45.

\section{REFERENCES}

\footnotetext{
${ }^{1}$ Corresponding author (Bo Ning).
} 
[1] P. Bedrossian, Forbidden Subgraph and Minimum Degree Conditons for Hamiltonicity (Ph.D. Thesis, Memphis State University, 1991).

[2] P. Bedrossian, G. Chen and R.H. Schelp, A generalization of Fan's condition for Hamiltonicity, pancyclicity, and Hamiltonian connectedness, Discrete Math. 115 (1993) 39-50. doi:10.1016/0012-365X(93)90476-A

[3] L.W. Beineke, Characterizations of derived graphs, J. Combin. Theory Ser. B 9 (1970) 129-135. doi:10.1016/S0021-9800(70)80019-9

[4] J.A. Bondy and U.S.R. Murty, Graph Theory (GTM 244, Springer, 2008).

[5] H.J. Broersma, Z. Ryjáček and I. Schiermeyer, Dirac's minimum degree condition restricted to claws, Discrete Math. 167/168 (1997) 155-166. doi:10.1016/S0012-365X(96)00224-5

[6] H.J. Broersma and H.J. Veldman, Restrictions on induced subgraphs ensuring hamiltonicity or pancyclicity of $K_{1,3}$-free graphs, in: Contemporary Methods in Graph Theory (BI Wissenschaftsverlag, Mannheim 1990) 181-194.

[7] J. Brousek, Minimal 2-connected non-Hamiltonian claw-free graphs, Discrete Math. 191 (1998) 57-64. doi:10.1016/S0012-365X(98)00093-4

[8] J. Brousek, Z. Ryjáček and O. Favaron, Forbidden subgraphs, hamiltonicity and closure in claw-free graphs, Discrete Math. 196 (1999) 29-50. doi:10.1016/S0012-365X(98)00334-3

[9] G. Chen, B. Wei and X. Zhang, Degree-light-free graphs and hamiltonian cycles, Graphs Combin. 17 (2001) 409-434. doi: $10.1007 /$ s003730170018

[10] R. Čada, Degree conditions on induced claws, Discrete Math. 308 (2008) 5622-5631. doi:10.1016/j.disc.2007.10.026

[11] G.A. Dirac, Some theorems on abstract graphs, Proc. London. Math. Soc. 2 (1952) 69-81.

doi:10.1112/plms/s3-2.1.69

[12] D. Duffus, M. Jacboson and R.J. Gould, Forbidden subgraphs and the Hamiltonian theme, in: The Theory and Applications of Graphs (Wiley, New York, 1981) $297-316$.

[13] G. Fan, New sufficient conditions for cycles in graphs, J. Combin. Theory Ser. B 37 (1984) 221-227. doi:10.1016/0095-8956(84)90054-6

[14] R.J. Faudree and R.J. Gould, Characterizing forbidden pairs for hamiltonian properties, Discrete Math. 173 (1997) 45-60. doi:10.1016/S0012-365X(96)00147-1 
[15] R.J. Faudree, R.J. Gould, Z. Ryjáček and I. Schiermeyer, Forbidden subgraphs and pancyclicity, Congr. Numer. 109 (1995) 13-32.

[16] R.J. Gould and M.S. Jacobson, Forbidden subgraphs and hamiltonian properties of graphs, Discrete Math. 42 (1982) 189-196. doi:10.1016/0012-365X(82)90216-3

[17] Z. Hu, A generalization of Fan's condition and forbidden subgraph conditions for hamiltonicity, Discrete Math. 196 (1999) 167-175. doi:10.1016/S0012-365X(98)00200-3

[18] B. Li, Z. Ryjáček, Y. Wang and S. Zhang, Pairs of heavy subgraphs for Hamiltonicity of 2-connected graphs, SIAM J. Discrete Math. 26 (2012) 1088-1103. doi: $10.1137 / 11084786 \mathrm{X}$

[19] G. Li, B. Wei and T. Gao, A structural method for Hamiltonian graphs, Australas. J. Combin. 11 (1995) 257-262.

[20] B. Ning and S. Zhang, Ore- and Fan-type heavy subgraphs for hamiltonicity of 2connected graphs, Discrete Math. 313 (2013) 1715-1725. doi:10.1016/j.disc.2013.04.023

[21] B. Ning, S. Zhang and B. Li, Solution to a problem on hamiltonicity of graphs under Ore- and Fan-type heavy subgraph conditions, Graphs Combin. 32 (2016) $1125-1135$.

doi:10.1007/s00373-015-1619-1

[22] O. Ore, Note on Hamilton circuit, Amer. Math. Monthly 67 (1960) 55.

[23] Z. Ryjáček, On a closure concept in claw-free graphs, J. Combin. Theory Ser. B 70 (1997) 217-224.

doi:10.1006/jctb.1996.1732

Received 22 June 2015

Revised 5 February 2016

Accepted 11 June 2016 